\title{
SONOGRAPHIC IDENTIFICATION OF UTERINE LEIOMYOMAS AND THEIR IMPACT ON FERTILITY
}

\author{
Anum Sarwar ${ }^{1}$, Syeda Khadija ${ }^{2}$, Muhammad Ateeb Ali Khan ${ }^{2}$, Nazeeha Waseem², and \\ Waqas Ali Ghouri ${ }^{2}$ \\ ${ }^{1}$ University of Lahore - Defence Road Campus \\ ${ }^{2}$ Affiliation not available
}

January 6, 2021

\begin{abstract}
ABSTRACT Context: Fibroids are the most common tumors of the female genital tract.fibroids are of benign growth develop in the muscular wall of the uterus composed primarily of smooth muscles. Fibroids are known by different other names like leiomyoma, leiomyomata, myoma and fibromyoma. It can be located in different part of uterus so named them according to their location in uterus. Fibroids vary in size from such small like a pea to as large to 10 inches. The relationship of fibroids with infertility is controversial to find an impact of uterine fibroids on infertility. Ultrasonography is a Gold Standard modality for identification of uterine fibroids. The purpose of this Retrospective study is to review fibroids and their different types and their impact on fertility. Fibroids effect females of every age group and their management are necessary for increase fertility outcomes. Objective: A systematic review is made with help of different retrospective studies published from time duration 2005 to 2020 on sonographic identification of uterine leiomyomas and their impact on fertility. Evidence acquisition: Relevant databases on uterine fibroid relation and impact on infertility was collected from MEDLINE, PubMed, Cochrane Library from 2005 to 2020. Randomised control trials, prospective observational studies, retrospective matched studies from well-defined registries are taken. Conclusion: Uterine fibroids having an impact on fertility its incidence increased with age.submucosal fibroids cause infertility, intramural fibroids impact on fertility is controversial while subserosal fibroids don't have an impact on fertility. Key words: Leiomyomas, Infertility, Abdominal Ultrasound, Transvaginal Ultrasound, Myomectomy, Fertility
\end{abstract}

\section{SONOGRAPHIC IDENTIFICATION OF UTERINE LEIOMYOMAS (FIBROIDS) AND THEIR IMPACT ON FERTILITY - A SYSTEMATIC REVIEW}

A Systematic Review Submitted by

\section{ANUM SARWAR (Author)}

MID, MS Diagnostic Ultrasound (scholar) 


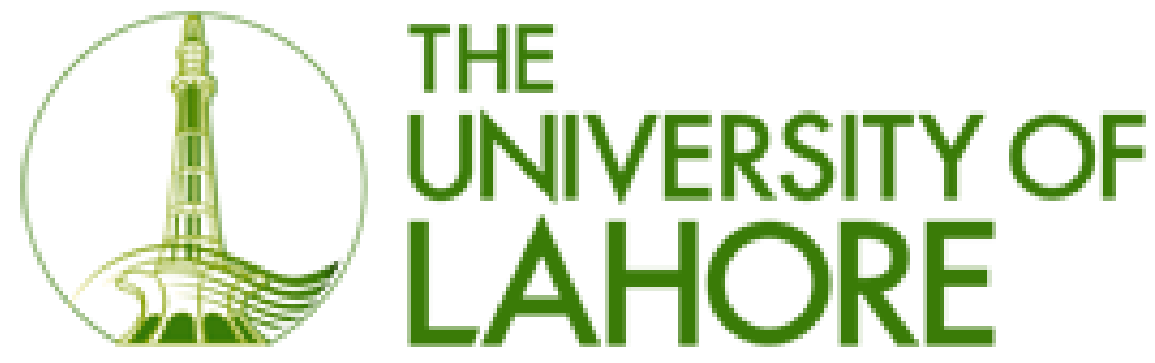

Student ID. 70033219

Dr. Syeda Khadija (Co Author)

$\mathrm{PhD}, \mathrm{MSDU}$ (Ultrasound)

Ateeb Ali Khan (Co Author)

Nazeeha Waseem (Co author)

Waqas Ali Ghouri(Co author)

University Institute of Radiological Sciences \& Medical Imaging Technology

Faculty of Allied Health Sciences

THE UNIVERSITY OF LAHORE

Session: (2020-2022)

ABSTRACT

Context: Fibroids are the most common tumors of the female genital tract.fibroids are of benign growth develop in the muscular wall of the uterus composed primarily of smooth muscles. Fibroids are known by different other names like leiomyoma, leiomyomata, myoma and fibromyoma. It can be located in different part of uterus so named them according to their location in uterus. Fibroids vary in size from such small like a pea to as large to 10 inches. The relationship of uterine fibroids with infertility and their impact on female fertility is still controversial. Ultrasonography is a Gold Standard modality for identification of uterine fibroids. The purpose of this Retrospective study is to review fibroids and their different types and their impact on fertility. Fibroids effect females of every age group and their management are necessary for increase fertility outcomes.

Aim: A systematic review is made with help of different retrospective studies published from time duration 2005 to 2020 on sonographic identification of uterine leiomyomas and their impact on fertility.

Methods: Relevant databases on uterine fibroid relation and impact on infertility was collected from MEDLINE, PubMed, Cochrane Library from 2005 to 2020. Randomised control trials, prospective observational studies, retrospective matched studies from well-defined registries are taken. Different systematic reviews by meta-analysis are taken.

Results: There are total 2451 abstracts and 480 full-text articles are studied and accessed. There were a high risk of bias and low-quality evidence but high-quality evidence support that the uterine leiomyomas impact on infertility is still controversial. A lot of studies conducted on it but all these studies provide 
controversial results. :eiomyomas impact on fertility but it seems to be that all leiomyomas don't cause infertility and different kind of interventions like myomectomy, uterine fibroids embolisation.

Conclusion: Uterine fibroids having an impact on fertility its incidence increased with age.submucosal fibroids cause infertility, intramural fibroids impact on fertility is controversial while subserosal fibroids don't have an impact on fertility.

Key words: Leiomyomas, Infertility, Abdominal Ultrasound, Transvaginal Ultrasound, Myomectomy, Fertility.

\section{INTRODUCTION:}

Uterine fibroids myomas or leiomyomas are the benign cell tumors of the females these are the most common benign growth tumors of female genital tract in reproductive age. ${ }^{1}$ Prevalence of uterine fibroids varies in females based on age, race and method which is used to diagnose it like Ultrasound, MRI etc. ${ }^{2}$ Fibroids are most commonly diagnosed in females come for investigation of infertility and often diagnosed with uterine fibroids. ${ }^{3}$ They are mostly asymptomatic and rarely found before menarche and on menopause and regress because they found to be hormonal sensitive and estrogen stimulates its growth. ${ }^{4,5}$ Submucosal fibroids are more hormonal responsive than subserosal. ${ }^{6} 7$ to 8 out of 10 females having fibroids in their lifetime. ${ }^{7}$ Fibroids induce chronic inflammation of myometrium and influence its contractility and a hindrance in implantation. ${ }^{8-12}$ The prevalence rate according to literature is 20 to $50 \%$ prevalence varies with age and race. The current study tells that prevalence of uterine fibroid increases with age and also tendency of getting pregnant become lower with increased age and more complicated with family history of fibroids and infertility. ${ }^{13}$ Majority of fibroids are asymptomatic. Symptoms which attributed to uterine fibroids are pelvic pain, tenderness, bloating, pelvic pressure, heavy menstrational bleeding and infertility. Females which become pregnant in presence of uterine fibroids it significantly effect on fibroid growth. ${ }^{14}$ Fibroids are made of bundle of smooth muscle cells. ${ }^{15}$ and arise in mutation in a single cell of myometrium and after mutation growth occurs via clonal expansion. ${ }^{16}$ Estrogen is responsible for the growth of fibroid and also there are other growth factors and progesterone which boosted its development. ${ }^{17-18}$ There are different mechanical factors that influence on fertility with respect to uterine fibroids like tubal ostia might be due to fibroid present in uterine cavity impact obstuction result in hindrance in sperm and embryo transport.physiopathological changes in myometrium and endometrium and pressure effect due to fibroid on endometrium elongation and distortion effect the window of implantation. ${ }^{19}$ In rare cases the uterine fibroids are of different types depends on their location and origin in uterus. Fibroids are found in myometrium. Submucosal fibroids are found into uterine cavity. Intramural fibroids found in the wall of uterus on ultrasound it seems to be a bulge on uterine wall. Fig 1 is showing a transabdominal ultrasound showing a pelvic scan of uterus in which a subserosal fibroid is evaluated. Subserosal fibroid cause sdetoriation in serosal wall of uterus and impact a lot on fertility. Its location is important because management and treatment of fibroid is based on its type and location. ${ }^{20}$ Grey scale ultrasonography is a Gold standard for the evaluation and diagnosis of uterine fibroids. First step to evaluate and diagnose uterine fibroid is ultrasound in which 2D, 3D, colour doppler and sonohysterography plays their additional part. 2D or grey scale ultrasound help to evaluate fibroids according to their sonographic features while colour doppler ultrasound help to access blood flow. It is helpful to differentiate it from malignant tumors. ${ }^{21}$ Different patterns of echogenicity of UF due to different internal composition and degenerations add to diagnostic confusion. Some sonographic features of uterine fibroids are variable appearance, hypoechoic or heterogeneous mass, distortion of external uterine contour, attenuation or shadowing without discrete mass, calcification, degenerative changes and necrosis. Intramural fibroids develop within the lining of uterus and expand inward, increasing the size of uterus, making it feel larger than the normal in gynaecological exam. Fig 2 showing a transabdominal image of uterine cavity showing 2 fibroids first image describing submucosal and second image is showing intramural fibroid. ${ }^{22}$ Uterine fibroids evaluation on ultrasound is accurate but some time misdiagnosed by other pelvic pathologies like adnexal masses. Subserosal pedenculated or exophytic fibroids which have small stalk misdiagnosed as adnexal mass. A laterally bulging fibroid giving appearance of symmetrically bilobed uterus and misdiagnosed as bicornuate uterus. ${ }^{23}$ Intestitial fibroids gave confusion 
about ectopic pregnancy. ${ }^{24}$ Different patterns of chogenicity of UF due to different internal composition and degenerations add to diagnostic confusion.

Some sonographic features of uterine fibroids are variable appearance, hypoechoic or heterogeneous mass, distortion of external uterine contour, attenuation or shadowing without discrete mass, calcification, degenerative changes and necrosis. ${ }^{24}$ According to different studies the uterine fibroids impact on infertility is controversial like subserosal fibroids have no impact on fertility. Mostly these are pedunculated having stalk cause abdominal pressure and distention. Intramural fibroid impact on fertility is not described completely it depends on their location and interfaces with endometrium so it may and may not cause infertility. Submucosal fibroid impact endometrial cavity and distort it having impact on fertility and cause infertility. .As shown in fig no 3 in a longitudnal image of uterus taken per abdominally showing a submucosal fibroid ${ }^{25}$ For fibroids different methods of management and treatment are used these days myomectomy is gold standard for UF and success rate is considerable in females with submucosal fibroids. Other methods include uterine artery embolisation (UAE), hysterectomy, hysteroscopic resection of Uterine fibroids, Endometrial ablation, MRI guided procedures. There are figure 4 ellaborating the uterine fibroid in uterus on different locations. 26

26

\begin{tabular}{|c|c|c|c|c|c|c|}
\hline Sr. No. & $\begin{array}{l}\text { Publication } \\
\text { Year }\end{array}$ & $\begin{array}{l}\text { Author } \\
\text { Name }\end{array}$ & Title & Variables & $\begin{array}{l}\text { Material \& } \\
\text { Methods }\end{array}$ & Results \\
\hline 1 & 2010 & $\begin{array}{l}\text { Heather } \\
\text { Cook }\end{array}$ & $\begin{array}{l}\text { The Impact } \\
\text { of Uterine } \\
\text { Leiomyomas } \\
\text { on Repro- } \\
\text { ductive } \\
\text { Outcomes }\end{array}$ & $\begin{array}{l}\text { Female Re- } \\
\text { production, } \\
\text { Leiomyomas }\end{array}$ & $\begin{array}{l}\text { Secondary } \\
\text { data } \\
\text { conducted } \\
\text { on impact } \\
\text { and } \\
\text { correlation } \\
\text { of } \\
\text { leiomyomas } \\
\text { on fertility }\end{array}$ & $\begin{array}{l}\text { Submucosal } \\
\text { and } \\
\text { intramural } \\
\text { myomas are } \\
\text { associated } \\
\text { with reduced } \\
\text { fertility. }\end{array}$ \\
\hline 2 & 2015 & $\begin{array}{l}\text { Belina } \\
\text { Carranza- } \\
\text { Mamane }\end{array}$ & $\begin{array}{l}\text { The } \\
\text { Management } \\
\text { of Uterine } \\
\text { Fibroids in } \\
\text { Women } \\
\text { With } \\
\text { Otherwise } \\
\text { Unexplained } \\
\text { Infertility }\end{array}$ & $\begin{array}{l}\text { Female } \\
\text { Infertility, } \\
\text { Fibroids } \\
\text { type }\end{array}$ & $\begin{array}{l}\text { Systematic } \\
\text { reviews on } \\
\text { literature } \\
\text { retrieved } \\
\text { from } \\
\text { PubMed, } \\
\text { MEDLINE } \\
\text { and consult } \\
\text { Cochrane } \\
\text { Library }\end{array}$ & $\begin{array}{l}\text { Submucosal } \\
\text { fibroids } \\
\text { seem to have } \\
\text { an impact, } \\
\text { whereas } \\
\text { subserosal } \\
\text { do not, } \\
\text { Intramural } \\
\text { fibroids } \\
\text { might have } \\
\text { an impact } \\
\text { on fertility }\end{array}$ \\
\hline
\end{tabular}




\begin{tabular}{|c|c|c|c|c|c|c|}
\hline Sr. No. & $\begin{array}{l}\text { Publication } \\
\text { Year }\end{array}$ & $\begin{array}{l}\text { Author } \\
\text { Name }\end{array}$ & Title & Variables & $\begin{array}{l}\text { Material \& } \\
\text { Methods }\end{array}$ & Results \\
\hline 3 & 2015 & $\begin{array}{l}\text { Leonidas I. } \\
\text { Zepiridis }\end{array}$ & $\begin{array}{l}\text { Infertility } \\
\text { and Uterine } \\
\text { Fibroids }\end{array}$ & $\begin{array}{l}\text { Uterine } \\
\text { Fibroid, } \\
\text { Infertility, } \\
\text { Fertility }\end{array}$ & $\begin{array}{l}\text { Meta } \\
\text { analysis on } \\
\text { hundrad of } \\
\text { researches } \\
\text { on } \\
\text { correlation } \\
\text { of fibroids } \\
\text { and } \\
\text { infertility }\end{array}$ & $\begin{array}{l}\text { Submucosal } \\
\text { of any size } \\
\text { and } \\
\text { intramural } \\
\text { uterine } \\
\text { fibroids of > } \\
4 \mathrm{~cm}, \\
\text { significantly } \\
\text { impairs } \\
\text { fertility and } \\
\text { IVF. The } \\
\text { presence of } \\
\text { subserosal } \\
\text { myomas has } \\
\text { little or no } \\
\text { effect on } \\
\text { fertility. }\end{array}$ \\
\hline 4 & 2016 & P. Purohit & $\begin{array}{l}\text { Fibroids and } \\
\text { Infertility }\end{array}$ & $\begin{array}{l}\text { Infertility, } \\
\text { Fibroids, } \\
\text { Myomec- } \\
\text { tomy, } \\
\text { Implanta- } \\
\text { tion, } \\
\text { Miscarriage, } \\
\text { Laparoscopy }\end{array}$ & $\begin{array}{l}\text { Observational } \\
\text { and review } \\
\text { based study } \\
\text { on } 42 \\
\text { articles }\end{array}$ & $\begin{array}{l}\text { Submucosal } \\
\text { fibroids } \\
\text { should be } \\
\text { treated } \\
\text { hysteroscop- } \\
\text { ically to } \\
\text { improve } \\
\text { conception } \\
\text { rates. } \\
\text { intramural } \\
\text { fibroids } \\
\text { management } \\
\text { should be in- } \\
\text { dividualised } \\
\text { on a case to } \\
\text { case basis, } \\
\text { whereas } \\
\text { subserosal } \\
\text { fibroids are } \\
\text { unlikely to } \\
\text { have any } \\
\text { major } \\
\text { impact on } \\
\text { fertility. }\end{array}$ \\
\hline
\end{tabular}




\begin{tabular}{|c|c|c|c|c|c|c|}
\hline Sr. No. & $\begin{array}{l}\text { Publication } \\
\text { Year }\end{array}$ & $\begin{array}{l}\text { Author } \\
\text { Name }\end{array}$ & Title & Variables & $\begin{array}{l}\text { Material \& } \\
\text { Methods }\end{array}$ & Results \\
\hline 5 & 2008 & $\begin{array}{l}\text { Aradhana } \\
\text { Khaund }\end{array}$ & $\begin{array}{l}\text { Impact of } \\
\text { fibroids on } \\
\text { reproductive } \\
\text { function }\end{array}$ & $\begin{array}{l}\text { Fibroids } \\
\text { Type, } \\
\text { Pregnancy, } \\
\text { Fertility, } \\
\text { Infertility }\end{array}$ & $\begin{array}{l}\text { Prospective } \\
\text { cohort } \\
\text { studies. }\end{array}$ & $\begin{array}{l}\text { Submucosal } \\
\text { and } \\
\text { intramural } \\
\text { fibroids } \\
\text { which } \\
\text { distorted } \\
\text { endometrial } \\
\text { cavity have } \\
\text { negative } \\
\text { effects on } \\
\text { ART .In } \\
\text { submucosal } \\
\text { fibroids my- } \\
\text { omectomy } \\
\text { have positive } \\
\text { impact. }\end{array}$ \\
\hline 6 & 2007 & $\begin{array}{l}\text { Neelanjana } \\
\text { Mukhopadhaya }\end{array}$ & $\begin{array}{l}\text { Uterine } \\
\text { fibroids: } \\
\text { impact on } \\
\text { fertility and } \\
\text { pregnancy } \\
\text { loss }\end{array}$ & $\begin{array}{l}\text { Uterine } \\
\text { fibroids, } \\
\text { Fertility, } \\
\text { Pregnancy }\end{array}$ & $\begin{array}{l}\text { Systematic } \\
\text { review on } \\
\text { previous } \\
\text { studies }\end{array}$ & $\begin{array}{l}\text { fibroids are } \\
\text { associated } \\
\text { with } \\
\text { infertility in } \\
5-10 \% \text { and } \\
\text { sole cause of } \\
\text { infer- } \\
\text { tility in } 2-3 \% \\
\text { women.submucos } \\
\text { and } \\
\text { intramural } \\
\text { are } \\
\text { associated } \\
\text { with } \\
\text { subfertility } \\
\text { and } \\
\text { subserosal } \\
\text { fibroids have } \\
\text { no impact } \\
\text { on fertility. }\end{array}$ \\
\hline 7 & 2011 & $\begin{array}{l}\text { Pankaj } \\
\text { Desai }\end{array}$ & $\begin{array}{l}\text { Fibroids, } \\
\text { Infertility } \\
\text { and Laparo- } \\
\text { scopic } \\
\text { Myomectomy }\end{array}$ & $\begin{array}{l}\text { Fibroid, La- } \\
\text { paroscopic } \\
\text { Myomec- } \\
\text { tomy, } \\
\text { infertility }\end{array}$ & $\begin{array}{l}\text { Articles } \\
\text { published } \\
\text { between } \\
1980 \text { and } \\
2010 \text { taken } \\
\text { from } \\
\text { Medline, } \\
\text { PubMed, } \\
\text { and } \\
\text { Cochrane } \\
\text { Databases }\end{array}$ & $\begin{array}{l}\text { Fertility } \\
\text { decreased in } \\
\text { women with } \\
\text { submucosal } \\
\text { fibroids, } \\
\text { Subserosal } \\
\text { fibroids do } \\
\text { not affect } \\
\text { fertility and } \\
\text { Intramural } \\
\text { fibroids } \\
\text { decrease } \\
\text { fertility. }\end{array}$ \\
\hline
\end{tabular}




\begin{tabular}{|c|c|c|c|c|c|c|}
\hline Sr. No. & $\begin{array}{l}\text { Publication } \\
\text { Year }\end{array}$ & $\begin{array}{l}\text { Author } \\
\text { Name }\end{array}$ & Title & Variables & $\begin{array}{l}\text { Material \& } \\
\text { Methods }\end{array}$ & Results \\
\hline 8 & 2007 & $\begin{array}{l}\text { Andrew W. } \\
\text { Horne }\end{array}$ & $\begin{array}{l}\text { The Effect } \\
\text { of Uterine } \\
\text { Fibroids on } \\
\text { Embryo } \\
\text { Implantation }\end{array}$ & $\begin{array}{l}\text { Fibroids, } \\
\text { Implanta- } \\
\text { tion, } \\
\text { Embryo }\end{array}$ & $\begin{array}{l}\text { Secondary } \\
\text { data of } \\
\text { previous } \\
\text { searches and } \\
\text { published } \\
\text { articles }\end{array}$ & $\begin{array}{l}\text { Due to } \\
\text { variation in } \\
\text { size ,location } \\
\text { and number } \\
\text { of fibroids } \\
\text { there is no } \\
\text { causal } \\
\text { relationship } \\
\text { between } \\
\text { fibroids and } \\
\text { failure of } \\
\text { embryo } \\
\text { implantation. }\end{array}$ \\
\hline 9 & 2005 & C. Benecke & $\begin{array}{l}\text { Effect of } \\
\text { Fibroids on } \\
\text { Fertility in } \\
\text { Patients } \\
\text { Undergoing } \\
\text { Assisted } \\
\text { Reproduction }\end{array}$ & $\begin{array}{l}\text { Fibroids, } \\
\text { Fertility, } \\
\text { ART }\end{array}$ & $\begin{array}{l}\text { Criteria } \\
\text { considered } \\
\text { pregnancy } \\
\text { data on in } \\
\text { vitro } \\
\text { fertilization, } \\
\text { intramural } \\
\text { myomata } \\
\text { with no } \\
\text { cavitary } \\
\text { distortion } \\
\text { and control } \\
\text { groups } \\
\text { without } \\
\text { myomas for } \\
\text { each patient } \\
\text { with a } \\
\text { myoma. }\end{array}$ & $\begin{array}{l}\text { There was a } \\
\text { significant } \\
\text { negative } \\
\text { impact on } \\
\text { implantation } \\
\text { rate in the } \\
\text { intramural } \\
\text { myomata } \\
\text { groups } \\
\text { versus the } \\
\text { control } \\
\text { groups, }\end{array}$ \\
\hline 10 & 2017 & $\begin{array}{l}\text { Rachel M. } \\
\text { Whynott }\end{array}$ & $\begin{array}{l}\text { The Effect } \\
\text { of Uterine } \\
\text { Fibroids on } \\
\text { Infertility: A } \\
\text { Systematic } \\
\text { Review }\end{array}$ & $\begin{array}{l}\text { Uterine } \\
\text { Fibroids, } \\
\text { Infertility }\end{array}$ & $\begin{array}{l}\text { Systematic } \\
\text { review by } \\
\text { meta- } \\
\text { analysis, } 20 \\
\text { electronic } \\
\text { search of the } \\
\text { PubMed } \\
\text { database } \\
\text { using the } \\
\text { keywords } \\
\text { "fibroid" and } \\
\text { "infertility" }\end{array}$ & $\begin{array}{l}\text { Submucosal } \\
\text { fibroids have } \\
\text { impact on } \\
\text { infertility. } \\
\text { Intramural } \\
\text { fibroids } \\
\text { greater than } \\
3 \text { cm are } \\
\text { also } \\
\text { implicated } \\
\text { in infertility } \\
\text { and } \\
\text { Subserosal } \\
\text { fibroids } \\
\text { generally do } \\
\text { not influence } \\
\text { a womans } \\
\text { fertility. }\end{array}$ \\
\hline
\end{tabular}




\begin{tabular}{|c|c|c|c|c|c|c|}
\hline Sr. No. & $\begin{array}{l}\text { Publication } \\
\text { Year }\end{array}$ & $\begin{array}{l}\text { Author } \\
\text { Name }\end{array}$ & Title & Variables & $\begin{array}{l}\text { Material \& } \\
\text { Methods }\end{array}$ & Results \\
\hline 11 & 2008 & $\begin{array}{l}\text { BARBARA } \\
\text { S. LEVY }\end{array}$ & $\begin{array}{l}\text { Modern } \\
\text { management } \\
\text { of uterine } \\
\text { fibroids }\end{array}$ & $\begin{array}{l}\text { Fibroids, } \\
\text { Myomectomy }\end{array}$ & $\begin{array}{l}\text { literature } \\
\text { review was } \\
\text { conducted } \\
\text { using } \\
\text { PubMed, } \\
\text { which } \\
\text { comprised } \\
\text { fibroids, } \\
\text { myomas, } \\
\text { mifepris- } \\
\text { tone, } \\
\text { myomectomy }\end{array}$ & $\begin{array}{l}\text { submucosal } \\
\text { fibroids have } \\
\text { impact on } \\
\text { fertility } \\
\text { because it } \\
\text { effects en- } \\
\text { dometrium } \\
\text { while } \\
\text { intramural } \\
\text { fibroids } \\
\text { impact on } \\
\text { fertility is } \\
\text { controversial. }\end{array}$ \\
\hline 12 & 2012 & $\begin{array}{l}\text { P. } \\
\text { Gambadauro }\end{array}$ & $\begin{array}{l}\text { Dealing with } \\
\text { uterine } \\
\text { fibroids in } \\
\text { reproductive } \\
\text { medicine }\end{array}$ & $\begin{array}{l}\text { Uterine } \\
\text { Fibroids, } \\
\text { Reproduc- } \\
\text { tive } \\
\text { medicines }\end{array}$ & $\begin{array}{l}\text { systematic } \\
\text { review on } \\
\text { uterine } \\
\text { fibroids are } \\
\text { an } \\
\text { increasingly } \\
\text { common } \\
\text { finding in } \\
\text { women } \\
\text { undergoing } \\
\text { assisted } \\
\text { reproduction }\end{array}$ & $\begin{array}{l}\text { submucosal } \\
\text { fibroids } \\
\text { interfere } \\
\text { with fertility } \\
\text {.intramural } \\
\text { fibroid if } \\
\text { influence en- } \\
\text { dometrium } \\
\text { than effect } \\
\text { fertility and } \\
\text { subserosal } \\
\text { fibroid effect } \\
\text { on fertility is } \\
\text { controversial. }\end{array}$ \\
\hline 13 & 2017 & $\begin{array}{l}\text { Marek } \\
\text { Lisiecki }\end{array}$ & $\begin{array}{l}\text { Fertility } \\
\text { impairment } \\
\text { associated } \\
\text { with uterine } \\
\text { fibroids - a } \\
\text { review of } \\
\text { literature }\end{array}$ & $\begin{array}{l}\text { Fertility, } \\
\text { Uterine } \\
\text { Fibroids }\end{array}$ & $\begin{array}{l}\text { comprehensive } \\
\text { review of } \\
\text { literature on } \\
\text { uterine } \\
\text { fibroids and } \\
\text { their } \\
\text { influence on } \\
\text { fertility } \\
\text { impairment }\end{array}$ & $\begin{array}{l}\text { submucosal } \\
\text { and } \\
\text { intramural } \\
\text { fibroids } \\
\text { which } \\
\text { distorted } \\
\text { endometrial } \\
\text { cavity } \\
\text { impact on } \\
\text { fertility . }\end{array}$ \\
\hline
\end{tabular}




\begin{tabular}{|c|c|c|c|c|c|c|}
\hline Sr. No. & $\begin{array}{l}\text { Publication } \\
\text { Year }\end{array}$ & $\begin{array}{l}\text { Author } \\
\text { Name }\end{array}$ & Title & Variables & $\begin{array}{l}\text { Material \& } \\
\text { Methods }\end{array}$ & Results \\
\hline 14 & 2009 & $\begin{array}{l}\text { Elizabeth A. } \\
\text { Pritts }\end{array}$ & $\begin{array}{l}\text { Fibroids and } \\
\text { infertility: } \\
\text { an updated } \\
\text { systematic } \\
\text { review of the } \\
\text { evidence }\end{array}$ & $\begin{array}{l}\text { Fertility, } \\
\text { Infertility }\end{array}$ & $\begin{array}{l}\text { Systematic } \\
\text { literature } \\
\text { review and } \\
\text { meta- } \\
\text { analysis of } \\
\text { existing } \\
\text { controlled } \\
\text { studies. } \\
\text { Private } \\
\text { center for } \\
\text { Reproduc- } \\
\text { tive } \\
\text { endocrinol- } \\
\text { ogy and } \\
\text { infertility. } \\
\text { Patient(s): } \\
\text { Women with } \\
\text { fibroids and } \\
\text { infertility. }\end{array}$ & $\begin{array}{l}\text { women with } \\
\text { subserosal } \\
\text { fibroids had } \\
\text { no difference } \\
\text { in their } \\
\text { fertility out- } \\
\text { comes.females } \\
\text { with } \\
\text { intramural } \\
\text { and } \\
\text { submucosal } \\
\text { fibroid } \\
\text { appear to } \\
\text { decrease } \\
\text { fertility. }\end{array}$ \\
\hline 15 & 2017 & $\begin{array}{l}\text { Ayman } \\
\text { Al-Hendy }\end{array}$ & $\begin{array}{l}\text { Uterine } \\
\text { Fibroids: } \\
\text { Burden and } \\
\text { Unmet } \\
\text { Medical Need }\end{array}$ & $\begin{array}{l}\text { Fertility, } \\
\text { Infertility, } \\
\text { Black women }\end{array}$ & $\begin{array}{l}\text { Cross sectional } \\
\text { study }\end{array}$ & $\begin{array}{l}\text { Uterine } \\
\text { fibroids } \\
\text { associated } \\
\text { with infertility } \\
\text { having high } \\
\text { incidence in } \\
\text { black women } \\
\text { and their } \\
\text { treatment for } \\
\text { fertility is cost } \\
\text { effective and } \\
\text { burden on } \\
\text { economic } \\
\text { condition of } \\
\text { couples. }\end{array}$ \\
\hline
\end{tabular}




\begin{tabular}{|c|c|c|c|c|c|c|}
\hline Sr. No. & $\begin{array}{l}\text { Publication } \\
\text { Year }\end{array}$ & $\begin{array}{l}\text { Author } \\
\text { Name }\end{array}$ & Title & Variables & $\begin{array}{l}\text { Material \& } \\
\text { Methods }\end{array}$ & Results \\
\hline 16 & 2015 & $\begin{array}{l}\text { Fabio } \\
\text { Parazzini }\end{array}$ & $\begin{array}{l}\text { Pregnancy } \\
\text { outcome and } \\
\text { uterine } \\
\text { fibroids }\end{array}$ & $\begin{array}{l}\text { Uterine } \\
\text { Fibroids, } \\
\text { Pregnancy } \\
\text { Outcome }\end{array}$ & $\begin{array}{l}\text { Observational } \\
\text { comparative } \\
\text { study on } \\
\text { efficacy of } \\
\text { different } \\
\text { modalities of } \\
\text { treatment of } \\
\text { intramural } \\
\text { fibroids } \\
\text { before } \\
\text { pregnancy } \\
\text { on } \\
\text { pregnancy } \\
\text { outcome }\end{array}$ & $\begin{array}{l}\text { Submucous } \\
\text { fibroids have } \\
\text { negative } \\
\text { impact on } \\
\text { pregnancy } \\
\text { outcome. } \\
\text { The role of } \\
\text { intramural } \\
\text { or subserous } \\
\text { fibroids is } \\
\text { less clear } \\
\text { but multiple } \\
\text { or large } \\
\text { fibroids have } \\
\text { likely a } \\
\text { negative role } \\
\text { on } \\
\text { pregnancy } \\
\text { outcome }\end{array}$ \\
\hline 17 & 2010 & $\begin{array}{l}\text { Tariqu } \\
\text { Salman }\end{array}$ & $\begin{array}{l}\text { Uterine } \\
\text { fibroids, } \\
\text { management } \\
\text { and effect on } \\
\text { fertility }\end{array}$ & $\begin{array}{l}\text { Uterine } \\
\text { fibroids, } \\
\text { Effect } \\
\text { Fertility }\end{array}$ & $\begin{array}{l}\text { Secondary } \\
\text { data }\end{array}$ & $\begin{array}{l}\text { Submucosal } \\
\text { fibroid and } \\
\text { intramural } \\
\text { fibroid effect } \\
\text { fertility. }\end{array}$ \\
\hline 18 & 2013 & $\begin{array}{l}\text { Elizabeth A. } \\
\text { Stewart }\end{array}$ & $\begin{array}{l}\text { The Burden } \\
\text { of Uterine } \\
\text { Fibroids for } \\
\text { African- } \\
\text { American } \\
\text { Women: } \\
\text { Results of a } \\
\text { National } \\
\text { Survey }\end{array}$ & $\begin{array}{l}\text { Uterine } \\
\text { Fibroids, } \\
\text { African- } \\
\text { American } \\
\text { women }\end{array}$ & $\begin{array}{l}\text { Comparative } \\
\text { study online } \\
\text { survey on } \\
\text { U.S women } \\
\text { age 29-59 } \\
\text { with symp- } \\
\text { tomatic } \\
\text { fibroids and } \\
\text { african } \\
\text { american } \\
\text { women. }\end{array}$ & $\begin{array}{l}\text { African- } \\
\text { American } \\
\text { women have } \\
\text { more severe } \\
\text { symptoms, } \\
\text { unique } \\
\text { concerns, } \\
\text { and different } \\
\text { information- } \\
\text { seeking } \\
\text { behavior for } \\
\text { fibroids. }\end{array}$ \\
\hline 19 & 2013 & $\begin{array}{l}\text { Bijan J. } \\
\text { Borah }\end{array}$ & $\begin{array}{l}\text { The impact } \\
\text { of uterine } \\
\text { leiomyomas: } \\
\text { a national } \\
\text { survey of } \\
\text { affected } \\
\text { women }\end{array}$ & $\begin{array}{l}\text { Hysterectomy, } \\
\text { Leiomyoma, } \\
\text { Quality of } \\
\text { Life, } \\
\text { Symptoms, } \\
\text { Uterine } \\
\text { Fibroids }\end{array}$ & $\begin{array}{l}\text { Comparative } \\
\text { study based } \\
\text { on national } \\
\text { survey aged } \\
\text { 29-59 years } \\
\text { with } \\
\text { self-reported } \\
\text { symp- } \\
\text { tomatic } \\
\text { uterine } \\
\text { leiomyomas }\end{array}$ & $\begin{array}{l}\text { Uterine } \\
\text { fibroids } \\
\text { cause more } \\
\text { morbidity in } \\
\text { females who } \\
\text { want to } \\
\text { conceive } \\
\text { specially } \\
\text { after age } 40 \text {. }\end{array}$ \\
\hline
\end{tabular}




\begin{tabular}{|c|c|c|c|c|c|c|}
\hline Sr. No. & $\begin{array}{l}\text { Publication } \\
\text { Year }\end{array}$ & $\begin{array}{l}\text { Author } \\
\text { Name }\end{array}$ & Title & Variables & $\begin{array}{l}\text { Material \& } \\
\text { Methods }\end{array}$ & Results \\
\hline 20 & 2013 & M. David & $\begin{array}{l}\text { Uterine } \\
\text { Fibroid Em- } \\
\text { bolisation - } \\
\text { Potential } \\
\text { Impact on } \\
\text { Fertility and } \\
\text { Pregnancy } \\
\text { Outcome }\end{array}$ & $\begin{array}{l}\text { Uterine } \\
\text { Fibroid, } \\
\text { Embolization }\end{array}$ & $\begin{array}{l}\text { Prospective } \\
\text { studies on } \\
\text { impact of } \\
\text { UAE } \\
\text { therapy on } \\
\text { fertility and } \\
\text { pregnancy } \\
\text { outcomes }\end{array}$ & $\begin{array}{l}\text { Fibroids } \\
\text { have } \\
\text { negative } \\
\text { influence on } \\
\text { fertility. so } \\
\text { females who } \\
\text { want to } \\
\text { conceive } \\
\text { with fibroids } \\
\text { goes for } \\
\text { uterine } \\
\text { fibroid } \\
\text { embolization } \\
\text { get less } \\
\text { satisfactory } \\
\text { results it } \\
\text { increase risk } \\
\text { of } \\
\text { spontaneous } \\
\text { abortion } \\
\text { than normal } \\
\text { pregnancy. }\end{array}$ \\
\hline 21 & 2011 & $\begin{array}{l}\text { Sotirios H. } \\
\text { Saravelos }\end{array}$ & $\begin{array}{l}\text { The } \\
\text { prevalence } \\
\text { and impact } \\
\text { of fibroids } \\
\text { and their } \\
\text { treatment } \\
\text { on the } \\
\text { outcome of } \\
\text { pregnancy in } \\
\text { women with } \\
\text { recurrent } \\
\text { miscarriage }\end{array}$ & $\begin{array}{l}\text { Fibroids, } \\
\text { Myomec- } \\
\text { tomy, } \\
\text { Recurrent } \\
\text { Miscarriage, } \\
\text { Pregnancy } \\
\text { Outcome }\end{array}$ & $\begin{array}{l}\text { Analysis of } \\
\text { retrospective } \\
\text { and } \\
\text { prospective } \\
\text { data }\end{array}$ & $\begin{array}{l}\text { Females } \\
\text { with uterine } \\
\text { fibroid } \\
\text { which } \\
\text { distorted the } \\
\text { cavity } \\
\text { undergoes } \\
\text { by } \\
\text { interventions } \\
\text { have } \\
\text { increased } \\
\text { rate of mid } \\
\text { term } \\
\text { miscarrages } \\
\text { as compare } \\
\text { to females in } \\
\text { which } \\
\text { fibroids } \\
\text { don't distort } \\
\text { the cavity. }\end{array}$ \\
\hline
\end{tabular}




\begin{tabular}{|c|c|c|c|c|c|c|}
\hline Sr. No. & $\begin{array}{l}\text { Publication } \\
\text { Year }\end{array}$ & $\begin{array}{l}\text { Author } \\
\text { Name }\end{array}$ & Title & Variables & $\begin{array}{l}\text { Material \& } \\
\text { Methods }\end{array}$ & Results \\
\hline 22 & 2014 & $\begin{array}{l}\text { Javier } \\
\text { Monleón }\end{array}$ & $\begin{array}{l}\text { Successful } \\
\text { Pregnancy } \\
\text { after } \\
\text { Treatment } \\
\text { with } \\
\text { Ulipristal } \\
\text { Acetate for } \\
\text { Uterine } \\
\text { Fibroids }\end{array}$ & $\begin{array}{l}\text { Fibroids, } \\
\text { Ulipristal } \\
\text { Acetate, } \\
\text { Pregnancy }\end{array}$ & $\begin{array}{l}\text { Case study } \\
\text { report }\end{array}$ & $\begin{array}{l}\text { A patient } \\
\text { with uterine } \\
\text { fibroid } \\
\text { introduce } \\
\text { ulipristal } \\
\text { acetate } \\
\text { result in } \\
\text { regression in } \\
\text { uterine } \\
\text { fibroid and } \\
\text { progressive } \\
\text { intrauterine } \\
\text { pregnancy } \\
\text { result in } \\
\text { successful } \\
\text { delivery. }\end{array}$ \\
\hline 23 & 2010 & $\begin{array}{l}\text { David L. } \\
\text { Olive }\end{array}$ & $\begin{array}{l}\text { Fibroids and } \\
\text { Reproduction }\end{array}$ & $\begin{array}{l}\text { Fibroids } \\
\text { Type, } \\
\text { Pregnancy, } \\
\text { Fertility, } \\
\text { Infertility }\end{array}$ & $\begin{array}{l}\text { Secondary } \\
\text { data }\end{array}$ & $\begin{array}{l}\text { Uterine } \\
\text { fibroids } \\
\text { impart in } \\
\text { infertil- } \\
\text { ity.submucosal } \\
\text { myoma } \\
\text { cause } \\
\text { infertility, } \\
\text { Intramural } \\
\text { fibroids may } \\
\text { decrease } \\
\text { fertility and } \\
\text { subserosal } \\
\text { having no } \\
\text { ethentic } \\
\text { evidence to } \\
\text { influence on } \\
\text { fertility. }\end{array}$ \\
\hline 24 & 2006 & $\begin{array}{l}\text { ERTAN } \\
\text { SARIDOGAN }\end{array}$ & $\begin{array}{l}\text { Endoscopic } \\
\text { management } \\
\text { of uterine } \\
\text { fibroids }\end{array}$ & $\begin{array}{l}\text { Fibroids, } \\
\text { Myomectomy }\end{array}$ & $\begin{array}{l}\text { Retrospective } \\
\text { study }\end{array}$ & $\begin{array}{l}\text { Submucosal } \\
\text { fibroids } \\
\text { cause } \\
\text { menstrual } \\
\text { disorders } \\
\text { and } \\
\text { subfertility } \\
\text { while impact } \\
\text { of } \\
\text { intramural } \\
\text { and sub } \\
\text { serosal } \\
\text { fibroids are } \\
\text { controversial. }\end{array}$ \\
\hline
\end{tabular}




\begin{tabular}{|c|c|c|c|c|c|c|}
\hline Sr. No. & $\begin{array}{l}\text { Publication } \\
\text { Year }\end{array}$ & $\begin{array}{l}\text { Author } \\
\text { Name }\end{array}$ & Title & Variables & $\begin{array}{l}\text { Material \& } \\
\text { Methods }\end{array}$ & Results \\
\hline 25 & 2008 & $\begin{array}{l}\text { Giovanna } \\
\text { Tropeano }\end{array}$ & $\begin{array}{l}\text { Non-surgical } \\
\text { management } \\
\text { of uterine } \\
\text { fibroids }\end{array}$ & $\begin{array}{l}\text { Embolization, } \\
\text { Fibroid, } \\
\text { Focused } \\
\text { Ultrasound, } \\
\text { Medical } \\
\text { Therapy }\end{array}$ & $\begin{array}{l}\text { PubMED, } \\
\text { MEDLIE, } \\
\text { Cochrane } \\
\text { library. }\end{array}$ & $\begin{array}{l}\text { Uterine } \\
\text { artery } \\
\text { embolization } \\
\text { results in } \\
\text { good effects } \\
\text { in fibroids } \\
\text { non surgical } \\
\text { management. }\end{array}$ \\
\hline 26 & 2007 & $\begin{array}{l}\text { William H. } \\
\text { Parker }\end{array}$ & $\begin{array}{l}\text { Etiology, } \\
\text { symptoma- } \\
\text { tology, and } \\
\text { diagnosis of } \\
\text { uterine } \\
\text { myomas }\end{array}$ & $\begin{array}{l}\text { Leiomyomas, } \\
\text { types, } \\
\text { Fertility, } \\
\text { Reproduction }\end{array}$ & $\begin{array}{l}\text { Retrospective } \\
\text { observa- } \\
\text { tional } \\
\text { study }\end{array}$ & $\begin{array}{l}\text { Regarding } \\
\text { to current } \\
\text { literature } \\
\text { leiomyomas } \\
\text { are not well } \\
\text { understood. }\end{array}$ \\
\hline 27 & 2011 & $\begin{array}{l}\text { Ben } \\
\text { KROON }\end{array}$ & $\begin{array}{l}\text { Fibroids in } \\
\text { infertility - } \\
\text { consensus } \\
\text { statement } \\
\text { from } \\
\text { ACCEPT }\end{array}$ & $\begin{array}{l}\text { Assisted Re- } \\
\text { productive } \\
\text { Technology, } \\
\text { Fibroids, } \\
\text { Infertility }\end{array}$ & $\begin{array}{l}\text { Trail } \\
\text { evidence } \\
\text { base study }\end{array}$ & $\begin{array}{l}\text { Subserosal } \\
\text { fibroid don't } \\
\text { influence on } \\
\text { fertility, } \\
\text { intramural } \\
\text { and } \\
\text { submucosal } \\
\text { fibroids may } \\
\text { associate } \\
\text { with } \\
\text { infertility. }\end{array}$ \\
\hline 28 & 2014 & $\begin{array}{l}\text { Aamir T } \\
\text { Khan }\end{array}$ & $\begin{array}{l}\text { Uterine } \\
\text { fibroids: } \\
\text { current } \\
\text { perspectives }\end{array}$ & $\begin{array}{l}\text { Leiomyomas, } \\
\text { Menorrhagia }\end{array}$ & $\begin{array}{l}\text { Cross- } \\
\text { sectional } \\
\text { analytical } \\
\text { study }\end{array}$ & $\begin{array}{l}\text { Uterine } \\
\text { fibroids are } \\
\text { associated } \\
\text { with } \\
\text { increased } \\
\text { morbidity } \\
\text { about } 40 \% \\
\text { impact on } \\
\text { infertility. }\end{array}$ \\
\hline 29 & 2007 & $\begin{array}{l}\text { E. } \\
\text { Somigliana }\end{array}$ & $\begin{array}{l}\text { Fibroids and } \\
\text { female re- } \\
\text { production: } \\
\text { a critical } \\
\text { analysis of } \\
\text { the evidence }\end{array}$ & $\begin{array}{l}\text { Uterine } \\
\text { Leiomyomas }\end{array}$ & $\begin{array}{l}\text { Observational } \\
\text { epidemiolog- } \\
\text { ical } \\
\text { studies }\end{array}$ & $\begin{array}{l}\text { Clinical } \\
\text { evidence } \\
\text { support the } \\
\text { opinion that } \\
\text { the } \\
\text { submucosal, } \\
\text { intramural } \\
\text { and } \\
\text { subserosal } \\
\text { fibroids } \\
\text { interfere } \\
\text { with fertility }\end{array}$ \\
\hline
\end{tabular}




\begin{tabular}{|c|c|c|c|c|c|c|}
\hline Sr. No. & $\begin{array}{l}\text { Publication } \\
\text { Year }\end{array}$ & $\begin{array}{l}\text { Author } \\
\text { Name }\end{array}$ & Title & Variables & $\begin{array}{l}\text { Material \& } \\
\text { Methods }\end{array}$ & Results \\
\hline 30 & 2014 & $\begin{array}{l}\text { Kristin Van } \\
\text { Heertum }\end{array}$ & $\begin{array}{l}\text { Uterine } \\
\text { fibroids } \\
\text { associated } \\
\text { with } \\
\text { infertility }\end{array}$ & $\begin{array}{l}\text { Uterine } \\
\text { Fibroids, } \\
\text { Fertility }\end{array}$ & $\begin{array}{l}\text { Review } \\
\text { study based } \\
\text { on } \\
\text { secondary } \\
\text { data }\end{array}$ & $\begin{array}{l}\text { Uterine } \\
\text { fibroid } \\
\text { incidence } \\
\text { increase } \\
\text { with age }\end{array}$ \\
\hline
\end{tabular}

\section{SYSTEMATIC REVIEW}

Uterine leiomyomas are benign tumors of female genital tract but its impact on fertility is still controversial .hundrads of studies conducted on their relationship.

A study was made on the impact of Uterine Leiomyomas on Reproductive Outcomes by Heather Cook in 2010 by using secondary data on correlation of fibroids and reproduction. They concluded that age and race having relation with fibroids as incidence of fibroids increased by age. Submucosal and intramural myomas are associated with reduced fertility. ${ }^{1}$

A study was conducted by Belina Carranza-Mamane on the topic of the Management of Uterine Fibroids in Women with otherwise Unexplained Infertility in 2015. Literature published by them was retrieved through PubMed, MEDLINE in November 2013 by Cochrane Library. Results of this study was confined to systematic reviews, Randomized observational, Clinical and Control trails. Authenticity of data was identified by using health technology agencies related websites clinical practice guidelines, Clinical trials and national and international medical societies. They concluded by this study that different type of fibroids shows different impact on fertility submucosal fibroid have impact on fertility whereas subserosal don't have an impact on fertility and intramural might have an impact on fertility. ${ }^{2}$

In 2015, Leonidas I. Zepiridis conducted a study on Infertility and Uterine Fibroids by using Secondary data based on meta-analysis made by different authors on hundred of researches on correlation of fibroids and infertility and they concluded on basis of available evidence that the submucosal fibroids of any size and intramural fibroids of $>4 \mathrm{~cm}$ significantly impairs fertility and IVF whereas subserosal fibroid has little or no effect on fertility. ${ }^{27}$

A research was conducted by P. Purohit in 2016 on fibroids and infertility .they used 42 articles and by their systematic review on basis of evidence he concluded that the relationship between fibroid and infertility is weak. According to him for getting conclusive results on correlation of fibroids with infertility and impact of uterine leiomyomas on fertility. The types and location of myomas and Impact on endometrium is important. Submucosal fibroids should be treated hysteroscopically. Intramural fibroids management vary case to case and subserosal fibroids don't have any major impact on infertility. ${ }^{28}$

A review based study was made by Aradhana Khaund in 2008 on Impact of fibroids on reproductive function. A large prospective study was conducted for evaluating the relationship between fibroids and infertility with comparison made with a control group of normal females and randomized trials on these control group was made and comparison of myomectomy versus other uterine sparing technique was made on these control groups. Submucosal and intramural fibroids which distort the endometrial cavity cause infertility and by ART miscarriages rate decreases and pregnancy rates successful. ${ }^{29}$

In 2007, Neelanjana Mukhopadhaya conducted research on Uterine Fibroids Impact on Fertility and Pregnancy Loss. It was a systematic review made by using previous studies and elaborate this data in form of charts, tables and summary. At present, according to previous data fibroids associated with infertility is 5-10 $\%$ by strong evidence submucosal fibroids are indeed associated with subfertility, and possibly miscarriage, and hysteroscopy resection is beneficial. Intramural fibroids impede successful outcomes of ART. Subserosal fibroids have no impact on pregnancy loss and fertility. ${ }^{30}$ 
Pankaj Desai conducted research on Fibroids in 2011, Infertility and Laparoscopic Myomectomy by using Medline, PubMed, and Cochrane Databases searched for articles published between 1980 and 2010. They concluded that females with submucosal fibroids having infertility and myomectomy are fruitful for fertility outcomes in these females. Subserosal fibroids have no impact on fertility and its removal is not necessary. Intramural fibroids appear to decrease fertility. Pregnancy rates in females with leiomyomatas are similar in females who gone under process of laparomtomy and management of UF through endoscope. ${ }^{31}$

Andrew Horne conducted research in 2007 on The Effect of Uterine Fibroids on Embryo Implantation by using secondary data of previous searches and published articles. In this research concluded that the causal relationship between fibroids and failure of embryo implantation is unclear. Unfortunately, due to variations in location, size, and number of fibroids and the presence of additional factors impact on fertility or infertility. This research was not conclusive and suggested that more fundamental research must be performed to detect the mechanisms of infertility, by comparing pregnancy rates of women with and without fibroids. ${ }^{32}$

A research review was made by C. Benecke on Effect of Fibroids on Fertility in Patients Undergoing Assisted Reproduction in 2005. In this review, articles were found by means of computerized Medline and Cochrane Library infertility and in vitro fertilization in human in 1990-2002. According to inclusion criteria pregnancy data on in vitro fertilization. One group of Intramural fibroids with no cavitary distortion and other control groups without myomas. According to their results a significant negative impact on implantation rate in the intramural myomata groups versus the control groups. ${ }^{33}$

Rachel M. Whynott made a systematic review in 2017 on The Effect of Uterine Fibroids on Infertility this systematic review was performed following the Preferred Reporting Items for Systematic Reviews and MetaAnalyses (PRISMA) guidelines. An electronic search of the PubMed database using the keywords "Fibroid" and "Infertility" was performed from 1996 until February 2017. Only English-language articles were included. A total of 558 articles were identified, or summary analyses of fertility outcomes were included. In this review, concluded that the uterine fibroids vary in size and shape and its presence affects fertility. Surgical interventions are beneficial as compare to non-invasive medical treatment. In management of uterine fibroids the myomectomy is Gold Standard, In management of infertility Submucosal fibroids should be treated. Intramural fibroids greater than $3 \mathrm{~cm}$ may cause infertility and Subserosal fibroids generally do not influence a woman's ability to get pregnant. ${ }^{34}$

Barbara S. Levy conducted a study on the Modern management of uterine fibroids in 2008, A detailed literature search was conducted using PubMed, which comprised the following search terms: uterine fibroids, asoprisnil, mifepristone, Gnt R hormone, laparotomy, laparoscopic myomectomy, hysteroscopy, myolysis, MRI ultrasound, cryomyolysis, UAE, D-UAE, MRI guided interstitial laser photocoagulation. Most of the references which are included and already published in 2018 and some material was obtained from online women's health resources provided by OBGYN.net, The Reproductive Science Center of the San Francisco and NIH \& CE. Numerous modalities for the management of uterine fibroids are available but many methods having some drawback so by considering all these things new modalities and methods were valuable. Many females who want to be pregnant while suffering from uterine fibroids for them uterine sparing approach is beneficial. The choice of used modality depends on female clinical presentation their need and approach which is used must be effective, safe and minimally invasive. ${ }^{35}$

In 2012, P. Gambadauro had a study on Dealing with Uterine Fibroids in Reproductive Medicine by using secondary data and get results that the impact and interference of uterine leiomyomas largely depend on their size and location submucosal fibroids have an impact on fertility and embryo implantation and its management are necessary for infertility treatment by considering their sign and symptoms. Intramural fibroids which distorted cavity impact on fertility and those who don't distort cavity their impact is controversial on fertility because distortion in cavity impact receptivity of endometrium and embryo implantation. No evidence in asymptomatic and infertile patients support the removal of subserosal fibroids. ${ }^{36}$

Marek Lisiecki made a literature review in 2017 on the title Fertility Impairment Associated with Uterine Fibroids - a review of literature. A literature review on UF and their influence on fertility impairment 
was made. PubMed and Google Scholar websites were used to find significant articles. Terms such as: "uterine fibroids", "fibroids", "leiomyomas", "myomas" were used in combination with "fertility", "infertility", "fertility impairment". The report was written and published in Polish and English language. According to existing literature, the relationship between infertility and uterine leiomyomas was described. According to them submucosal and intramural fibroid have an impact on fertility and receptivity and gene expression of endometrium specially submucosal fibroids which distort endometrial cavity.there was a study gap about intramural fibroid and they recommended further study on intramural fibroids impact on fertility. ${ }^{37}$

Elizabeth A. Pritts made a systematic review in 2009 on the topic of Fibroids and infertility: An updated Systematic Review of the Evidence. This systematic literature review and meta-analysis of existing controlled studies on private centre for reproductive endocrinology and infertility. Patients were women with fibroids and infertility and technique and study design was a systematic literature review, raw data extraction and data analysis. According to this evidence-based strong study women with subserosal fibroids have no impact on their fertility as compare to females with infertility and no myomas. Females with intramural fibroids having an impact on their fertility with less fertility and increased pregnancy loss but quality and authenticity was poor. Submucosal fibroids have an impact on fertility and after myomectomy its fertility outcomes increases. ${ }^{38}$

Ayman Al-Hendy made a research on Uterine Fibroids: Burden and Unmet Medical Needs in 2017 by using previous data review and concluded that Uterine fibroids affect a large segment of the population and can negatively impact on their daily routine and quality of living and result in o severe occupational and economic costs in effected females the rate of sufferance with uterine fibroids are more in black women and treatment of UF may cause temporary or permanent loss of fertility but with limited success with surgical and pharmacologic treatments are pursued. ${ }^{39}$

Fabio Parazzini conducted a research on Pregnancy Outcome and Uterine Fibroids in 2015. Large randomized trials were planned in order to compare the efficacy of different modalities of treatment of intramural fibroids before pregnancy on pregnancy outcome and got results that In conclusion clinical experiences and some observational studies suggests that submucosal fibroids have a negative impact on pregnancy outcome. The role of intramural or subserous fibroids is less clear but multiple or large fibroids have likely a negative role on pregnancy outcome. ${ }^{40}$

Tariqu Salman made a conclusive study on Uterine fibroids, Management and Effect on fertility in 2010 by using previous articles on fibroids and fertility relation and concluded that submucosal fibroids having no impact on fertility and intramural fibroids also doesn't impact fertility but it's controversial without strong evidence after studying the results of IV fertilisation there are no strong evidences that submucosal and intramural fibroids are associated with lower implantation, pregnancy and birth rates for fibroids more than $5 \mathrm{~cm}$ and distort endometrial cavity needs management of uterine fibroids after considering safety and efficacy. ${ }^{41}$

Elizabeth A. Stewart made a study in 2013 on The Burden of Uterine Fibroids for African American Women: Results of a National Survey bu using An online survey and concluded that as compare to American white women the African American women having unique concerns redarding fibroid behaviour, more sever symptoms, and unique different information regarding UF behaviour. ${ }^{42}$

Bijan J. Borah conducted a study in 2013 on the impact of uterine leiomyomas: A National Survey of affected Women. This comparative study was based on national survey aged 29-59 years with self-reported symptomatic uterine leiomyomas. According to their research results, uterine fibroids cause morbidity in females who want to conceive specially in age 40. it's supported the evidence that age impact fertility with complication of myomas. ${ }^{43}$

Javier Monleón make a case report study in 2014 on topic Successful Pregnancy after Treatment with Ulipristal Acetate for Uterine Fibroids by using variables, ulipristal acetate, pregnancy. Patient with uterine fibroid introduce ulipristal acetate result in a regression in uterine fibroid and progressive intrauterine pregnancy result in successful delivery. ${ }^{44}$ 
David L. Olive make a study in 2010 on Fibroids and Reproduction by using secondary data and concluded that uterine fibroids impact on infertility. Submucosal myoma cause infertility, intramural fibroids may decrease fertility and subserosal having no authentic evidence to influence on fertility. ${ }^{25}$

M. David made research in 2013 on Uterine Fibroid Embolisation - Potential Impact on Fertility and Pregnancy Outcomes by previous literature reviews they concluded that UAE should not be a treatment of choice for women who want to conceive there are currently very few prospective studies with results backed by sufficient evidence on impact of UAE therapy on fertility and pregnancy outcomes on basis of their literature review, the following recommendations are proposed: the existing evidence recommend that the females with myomas want to conceive operative myomectomy should be procedure of choice as compared to UAE prove fertility. The case series and studies show that UAE significantly increases the risk of spontaneous abortion additional investigations will be needed to understand the causes of the observe. ${ }^{45}$

Sotirios H. Saravelos made a study in 2011 on The prevalence and impact of fibroids and their treatment on the outcome of pregnancy in women with recurrent miscarriages couples were investigated according to established protocoles by using TV ultrasound and hysteroscopy and dignosed UF. Two study groups were subsequently examined: women with cavity-distorting fibroids who underwent surgery and women with fibroids not distorting the cavity who did not undergo any intervention. They concluded that females with uterine fibroids which distorted the cavity undergoes by interventions have increased rate of midterm miscarriages as compared to females in which fibroids don't distort the cavity. ${ }^{46}$

Ertan Saridoganmade research in 2006 on endoscopic management of uterine fibroids by using secondary data and concluded that Endoscopic management of uterine fibroids in women both with menstrual disorders and/or subfertility has become an established and widely used technique in the last few decades. Hysteroscopic myomectomy is now the standard treatment for the majority of women with submucosal fibroids. Laparoscopic myomectomy is suitable for a selected group of women with intramural and subserosal fibroids but requires considerable experience in advanced laparoscopic surgery. ${ }^{47}$

Giovanna Tropeano made a study in 2008 on Non-Surgical Management of Uterine Fibroids by using PubMed, Cochrane and Embase were searched up to December 2007. Different studies having good quality shows that UAE in symptomatic patients with fibroid having effectivness and safe procedure. In symptomatic patients newer treatments are still investigational. ${ }^{48}$

William H. parker made a literature review on Etiology, Symptomatology and Diagnosis of Uterine Myomas in 2007 by using a Literature review of 220 articles pertaining to uterine myomas and concluded that a summary of the available literature regarding the biology, aetiology, symptomatology and diagnosis of myomas shows that they are still not well understood, much has been learned about uterine myomas. ${ }^{49}$

Ben Kroon made research in 2011 on Fibroids in infertility consensus statement from ACCEPT by using previous articles and data according to this review Fibroids occur commonly in women of the reproductive age group. The impact of fibroid on fertility depends on their location and endometrial interface and other factors like age, family history etc.subserosal fibroids don't have a significant effect on fertility, and thus removal is generally under taken for symptomatic reasons only. IM fibroids may be associated with reduced fertility and an increased MR; however, there is insufficient evidence to inform whether myomectomy for IM fibroids improves fertility outcomes. SM fibroids are associated with reduced fertility and an increased MR, and myomectomy for SM fibroids appears likely to improve fertility outcomes. The relative effect of multiple or different sized fibroids on fertility outcomes is uncertain, as is the relative usefulness of myomectomy in these situations. ${ }^{50}$

Aamir T Khan made a review in 2014 on Uterine fibroids: current perspectives by using secondary data by review of 205 articles. They got brief results that although they are essentially benign, uterine fibroids are associated with significant morbidity to nearly $40 \%$ of women during their reproductive years and sometimes even after menopause.With more and more patients demanding a nonsurgical approach to their symptoms, there is a developing market for selective estrogen and especially progesterone receptor modulators, and exploring the role of vit D, Green tea and aromatase inhibitors. when the only surgical approach for fibroids 
was offering a myomectomy or hysterectomy. The concept of vascular embolization has altered the playing field and allowed women to preserve their fertility status without resorting to surgery. Minimally invasive management of fibroids has been even further enriched by the development and introduction. ${ }^{51}$

E. Somigliana made a review in 2007 on Fibroids and Female Reproduction: a critical analysis of the evidence by using secondary data and got conclusive brief results Epidemiological evidence on Although more limited, some evidence also supports an impact of the number and the dimension of the lesions. Clear guidelines for the management of fibroids in infertile women is however difficult due to the lack of large randomized trials aimed to evaluate which patients may benefit from surgery. I got conclusion that the submucosal, intramural and subserosal fibroids interfere with fertility We believe that this view is somehow simplistic and we suggest to adopt a comprehensive and personalized approach in the decision-making process to identify the best option for the woman. At least four points have to be considered: (i) the age of the woman; (ii) the location. ${ }^{52}$

In 2014, Kristin Van Heertum made a review-based study on uterine fibroids associated with infertility and concluded that uterine fibroids incidence increases with age. 53

\section{CONCLUSION:}

Leiomyomas are highly prevalent and vary by age and race with an incidence in females of every age group but increase with increased age. Fibroids in pregnancy are also very common. The impact of uterine fibroid on fertility depends on their type, size and location. Most commonly with high impact factor submucosal fibroid impede implantation and cause infertility. Myomectomy improves fertility outcomes and recommended for females want to conceive relation of intramural fibroids with infertility is controversial it may and may not impact on fertility depends on their interface with an endometrial cavity if it invades endometrial cavity may cause infertility and if cavity is intact than there is no impact on fertility. The subserosal fibroids don't impact on fertility.

\section{RECOMMENDATIONS:}

Different management and treatment methods are valid and implemented in medical gynaecological practice. There should be an authentic research work on invasive and non-invasive methods of treatment for UF for their type.

\section{ACKNOWLEDGEMENT:}

In the name of Allah, the most beneficent andmerciful. First and all praises be Allah, the Almighty, and the Glorious who has bestowed upon the faculties of thinking, searching and learning. I would like to express my gratitude to Mr Ateeb Ali Khan for their technical assistance.

\section{CONFLICT OF INTEREST:}

I Anum Sarwar declares that there is no conflict of interest.

\section{DISCLOURE OF INTEREST:}

I disclose that during this research we don't get any financial, personal and political financial aid. There was an intellectual interest to add up our role in medical knowledge.

\section{ETHICAL STATEMENT:}

I stated that during this study all ethical parameters are fulfilled and there is no voilation of human and animals right was made.

\section{FUNDING STATEMENT:}

I stated that we don't get any funding from any source.

\section{REFERENCES}


[1] Heather Cook, Mohammad Ezzati, James H. Segars, Desiree McCarthy; "The Impact of Uterine Leiomyomas on Reproductive Outcomes"; Minerva Ginecol.; 2010; 62(3), pp. 225-236

[2] Belina Carranza-Mamane, Sherbrooke, Jon Havelock, Vancouver BC, Robert Hemmings, Montreal; "The Management of Uterine Fibroids in Women With Otherwise Unexplained Infertility"; SOGC Clinical Practice Guideline; 2015; No-321, pp. 2787-285

[3] Katarina Englund, Agneta Blanck, Inger Gustavsson, Ulrika Lundkvist, Peter Sjoblom, Allan Norgren, Bo Lindblom; "Sex Steroid Receptors in Human Myometrium and Fibroids: Changes during the Menstrual Cycle and Gonadotropin-Releasing Hormone Treatment"; The Journal of Clinical Endocrinology \& Metabolism; 1998; Vol-83, Iss-11, pp. 4092-4096

[4] Edward E.Wallach, Veasy C.Buttram, Robert C.Reiter; "Uterine leiomyomata: etiology, symptomatology, and management"; Fertility and Sterility; 1981; Vol-36, Iss-4, pp. 433-445

[5] Erica E.Marsh, Serdar E.Bulun; "Steroid Hormones and Leiomyomas"; Obstetrics and Gynecology Clinics of North America; 2006; Vol-33, Iss-1, pp. 59-67

[6] Mario Marugo Michele Centonze Donatella Bernasconi Laura Fazzuoli Silvia Berta Giulio Giordano; "Estrogen and Progesterone Receptors in Uterine Leiomyomas"; Acta Obstetricia et Gynecologica; 1989; Vol-68, Iss-8, pp. 731-735

[7] Serdar E. Bulun; "Uterine Fibroids"; The New England Journal of Medicine; 2013; Vol-369(14), pp. $1344-1355$

[8] PA Richards, PDG Richards, AJ Tiltman; "The ultrastructure of fibromyomatous myometrium and its relationship to infertility"; Human Reproduction Update; 1998; Vol-4, Iss-5, pp. 520-525

[9] Beth W Rackow, Hugh S Taylor; "Submucosal uterine leiomyomas have a global effect on molecular determinants of endometrial receptivity"; Fertility and Sterility; 2010; Vol-93, Iss-6, pp. 2027-2034

[10] Aki Kido, Susan M Ascher, Keiko Kishimoto, Winnie Hahn, Reena C Jha, Kaori Togashi, James B Spies; "Comparison of uterine peristalsis before and after uterine artery embolization at 3-T MRI"; AJR Am J Roentgenology; 2011; Vol-196, No-6, pp. 1431-1435

[11] Osamu Yoshino, Osamu Nishii, Yutaka Osuga, Hisanori Asada, Shigeo Okuda, Makoto Orisaka, Masaaki Hori, Toshihiro Fujiwara, Toshihiko Hayashi; "Myomectomy decreases abnormal uterine peristalsis and increases pregnancy rate"; The Journal of Minimally Invasive Gynecology; 2012; Vol-19, Iss-1, pp. 63-67

[12] O Yoshino, T Hayashi, Y Osuga, M Orisaka, H Asada, S Okuda, M Hori, M Furuya, H Onuki, Y Sadoshima, H Hiroi, T Fujiwara, F Kotsuji, Y Yoshimura, O Nishii, Y Taketani; "Decreased pregnancy rate is linked to abnormal uterine peristalsis caused by intramural fibroids"; Human Reproduction Update; 2010; Vol-25, Iss-10, pp. 2475-2479

[13] Maria Luisa Casini, Federica Rossi, Riccardo Agostini, Vittorio Unfer; "Effects of the position of fibroids on fertility"; Gynecological Endocrinology; 2006; Vol-22, Iss-2, pp. 106-109

[14] Dr Elizabeth A Stewart; "Uterine fibroids"; E A Stewart; 2001; Vol-357, Iss-9295, pp. 293-298

[15] D E Townsend, R S Sparkes, M C Baluda, G McClelland; "Unicellular histogenesis of uterine leiomyomas as determined by electrophoresis by glucose-6-phosphate dehydrogenase"; American Journal of Obstetrics \& Gynecology; 1970; Vol-107, Iss-8, pp. 1168-1173

[16] M S Rein, A J Friedman, R L Barbieri, K Pavelka, J A Fletcher, C C Morton; "Cytogenetic abnormalities in uterine leiomyomata"; Obstet Gynecol; 1991; Vol-77(6), pp. 923-6

[17] D Linder, S M Gartler; "Glucose-6-phosphate dehydrogenase mosaicism: utilization as a cell marker in the study of leiomyomas"; Science; 1965; Vol-150, Iss-3692, pp. 67-69 
[18] R D Mashal, M L Fejzo, A J Friedman, N Mitchner, R A Nowak, M S Rein, C C Morton, J Sklar; "Analysis of androgen receptor DNA reveals the independent clonal origins of uterine leiomyomata and the secondary nature of cytogenetic aberrations in the development of leiomyomata"; Genes Chromosomes Cancer; 1994; Vol-11, Iss-1, pp. 1-6

[19] L Deligdish, M Loewenthal; "Endometrial changes associated with myomata of the uterus"; Journal of Clinical Pathology; 1970; Vol-23, Iss-8, pp. 676-680

[20] Elizabeth A Stewart; "Uterine fibroids"; The Lancet; 2001; Vol-357, Iss-9252, pp. 293-298

[21] Oksana H. Baltarowich, Alfred B. Kurtz, Rebecca G. Pennell, Laurence Needleman, Maria M. Vilaro, Barry B. Goldberg; "Pitfalls in the Sonographic Diagnosis of Uterine Fibroids"; American Journal of Roentgenology; 1988; Vol-151, Iss-4, pp. 725-728

[22] Sabrina Q. Rashid, Yi-Hong Chou, Chui-Mei Tiu; "Ultrasonography of Uterine Leiomyomas"; Journal of Medical Ultrasound; 2016; Vol-24, Iss-1, pp. 3-12

[23] Mary Graham, Peter L. Cooperberg; "Ultrasound diagnosis of interstitial pregnancy: findings and pitfalls"; Journal of Clinical Ultrasound; 1979; Vol-7, Iss-6, pp. 433-437

[24] V Persaud, P D Arjoon; "Uterine leiomyoma. Incidence of degenerative change and a correlation of associated symptoms"; Journal of Clinical Ultrasound; 1970; Vol-35(3), pp. 432-6

[25] David L. Olive, Elizabeth A. Pritts,; "Fibroids and Reproduction"; Seminars In Reproductive Medicine; 2010; Vol-28, Num-3, pp. 218-227

[26] Fascilla FD, Cramarossa P, Cannone R, Olivieri C, Vimercati A, Exacoustos C; "Ultrasound diagnosis of uterine myomas"; Minerva Ginecologica; 2016; Vol-68(3), pp. 297-312

[27] Leonidas I. Zepiridis, Grigoris F. Grimbizis, Basil C. Tarlatzis; "Infertility and Uterine Fibroids"; Best Practice \& Research Clinical Obstetrics \& Gynaecology; 2015; S1521-6934(15)00235-7, pp. 1-16

[28] P. Purohit, K. Vigneswaran; "Fibroids and Infertility"; Curr Obstet Gynecol Rep; 2016; DOI 10.1007/s13669-016-0162-2, pp. 81-88

[29] Aradhana Khaund, Aradhana Khaund; "Impact of fibroids on reproductive function"; Best Practice \& Research Clinical Obstetrics and Gynaecology; 2008; Vol-22, No-4, pp. 749-760

[30] Neelanjana Mukhopadhaya, Grace Pokuah Asante, Isaac T Manyonda; "Uterine fibroids: impact on fertility and pregnancy loss"; Obstetrics, Gynaecology and Reproductive Medicine ; 2007; 17:11, pp. 311-317

[31] Pankaj Desai, Purvi Patel; "Fibroids, Infertility and Laparoscopic Myomectomy"; Journal of Gynecological Endoscopy and Surgery; 2011; Vol-2, Issue-1, pp. 36-42

[32] Andrew W. Horne, Hilary O.D. Critchley; "The Effect of Uterine Fibroids on Embryo Implantation"; Seminars In Reproductive Medicine; 2007; Vol-25, Num-6, pp. 483-489

[33] C. Benecke, T.F. Kruger, T.I. Siebert, J.P. Van der Merwe, D.W. Steyn; 'Effect of Fibroids on Fertility in Patients Undergoing Assisted Reproduction"; Gynecol Obstet Invest; 2005; DOI: 10.1159/000084513, 59: pp. $225-230$

[34] Rachel M. Whynott, Kamaria C. Cayton Vaught, James H. Segars; "The Effect of Uterine Fibroids on Infertility: A Systematic Review"; Seminars in Reproductive Medicine; 2017; Vol-35, Num-6, pp. 523-532

[35] Barbara S. Levy; "Modern management of uterine fibroids"; Acta Obstetricia et Gynecologica; 2008; DOI: $10.1080 / 00016340802146912,87:$ pp. $812-823$

[36] P. Gambadauro; "Dealing with uterine fibroids in reproductive medicine"; Journal of Obstetrics and Gynaecology; 2012; DOI: 10.3109/01443615.2011.644357, 32: pp. 210-216 
[37] Marek Lisiecki, Maciej Paszkowski, Sławomir Woźniak; "Fertility impairment associated with uterine fibroids - a review of literature"; Menopause Review/Przegląd Menopauzalny; 2017; DOI: https://doi.org/10.5114/pm.2017.72759, 16(4): pp. 137-140

[38] Elizabeth A. Pritts, William H. Parker,David L. Olive; 'Fibroids and infertility: an updated systematic review of the evidence"; American Society for Reproductive Medicine; 2009; Vol. 91, No. 4, pp. 1215-1223

[39] Ayman Al-Hendy, Evan Robert Myers, Elizabeth Stewart; "Uterine Fibroids: Burden and Unmet Medical Need"; Seminars in Reproductive Medicine; 2017; Vol-35, No-6, pp. 473-480

[40] Fabio Parazzini, Dr. Luca Tozzi, Stefano Bianchi; "Pregnancy outcome and uterine fibroids"; Best Practice \& Research Clinical Obstetrics \& Gynaecology; 2015; DOI: 10.1016/j.bpobgyn.2015.11.017, pp. $1-26$

[41] Tariqu Salman, Colin Davis; "Uterine fibroids, management and effect on fertility"; Current Opinion in Obstetrics and Gynecology ; 2010; DOI:10.1097/GCO.0b013e32833d3606, 22: pp. 295-303

[42] Elizabeth A. Stewart, Wanda K. Nicholson, Linda Bradley, Bijan J. Borah; "The Burden of Uterine Fibroids for African-American Women: Results of a National Survey"; Journal Of Women's Health; 2013; Vol-22, Num-10, pp. 807-816

[43] Bijan J. Borah, Wanda K. Nicholson, Linda Bradley, Elizabeth A. Stewart; "The impact of uterine leiomyomas: a national survey of affected women"; American Journal of Obstetrics \& Gynecology; 2013; DOI:10.1016/j.ajog.2013.07.017, 209: pp. 319.e1-20

[44] Javier Monleón, Alicia Martínez-Varea, Daniela Galliano, and Antonio Pellicer; "Successful Pregnancy after Treatment with Ulipristal Acetate for Uterine Fibroids"; Hindawi Publishing Corporation; 2014; Vol2014, dx.doi.org/10.1155/2014/314587, pp. 1-3

[45] M. David, T. Kröncke; 'Uterine Fibroid Embolisation - Potential Impact on Fertility and Pregnancy Outcome"; Geburtsh Frauenheilk ; 2013; DOI:10.1055/s-0032-1328318, 73: pp. 247-255

[46] Sotirios H. Saravelos, Junhao Yan, Hassan Rehmani, Tin-Chiu Li; "The prevalence and impact of fibroids and their treatment on the outcome of pregnancy in women with recurrent miscarriage"; Human Reproduction Update; 2011; Vol-26, No-12, pp. 3274-3279

[47] Ertan Saridogan \& Alfred Cutner; "Endoscopic management of uterine fibroids"; Informa Healthcare Human Fertility; 2006; Vol-9(4), pp. 201 - 208

[48] Giovanna Tropeano, Sonia Amoroso and Giovanni Scambia; "Non-surgical management of uterine fibroids"; Human Reproduction Update; 2008; Vol-14, No-3, pp. 259-274

[49] William H. Parker; "Etiology, symptomatology, and diagnosis of uterine myomas"; American Society for Reproductive Medicine; 2007; Vol-87, No-4, pp.725-736

[50] Ben Kroon, Neil Johnson, Michael Chapman, Anusch Yazdani, Roger Hart; "Fibroids in infertility consensus statement from ACCEPT"; Australian and New Zealand Journal of Obstetrics and Gynaecology; 2011; Vol-51, pp. 289-295

[51] Aamir T Khan, Manjeet Shehmar, Janesh K Gupta; "Uterine fibroids: current perspectives"; International Journal of Women's Health; 2014; Vol-20, No. 14:6, pp. 95-114

[52] E. Somigliana, P. Vercellini, R. Daguati, R. Pasin, O. De Giorgi and P.G. Crosignani; "Fibroids and female reproduction: a critical analysis of the evidence"; Human Reproduction Update; 2007; Vol-13, No-5, pp. $465-476$

[53] Kristin Van Heertum, Larry Barmat; "Uterine fibroids associated with infertility"; Journal Of W omen's Health; 2014; Vol-10(6), pp. 645-653

\section{FIGURES:}


Fig 1:A transabdominal ultrasound showing a pelvic scan of uterus in which a subserosal fibroid is evaluated.

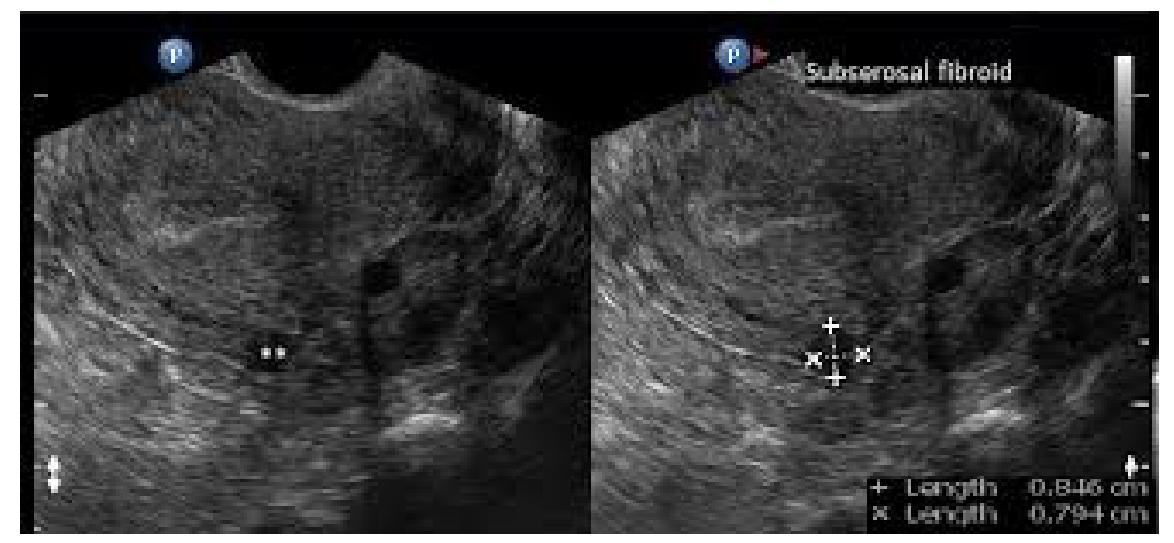

fig 2:a transabdominal image of uterine cavity is showing 2 fibroids a submucosal and intramural fibroids.

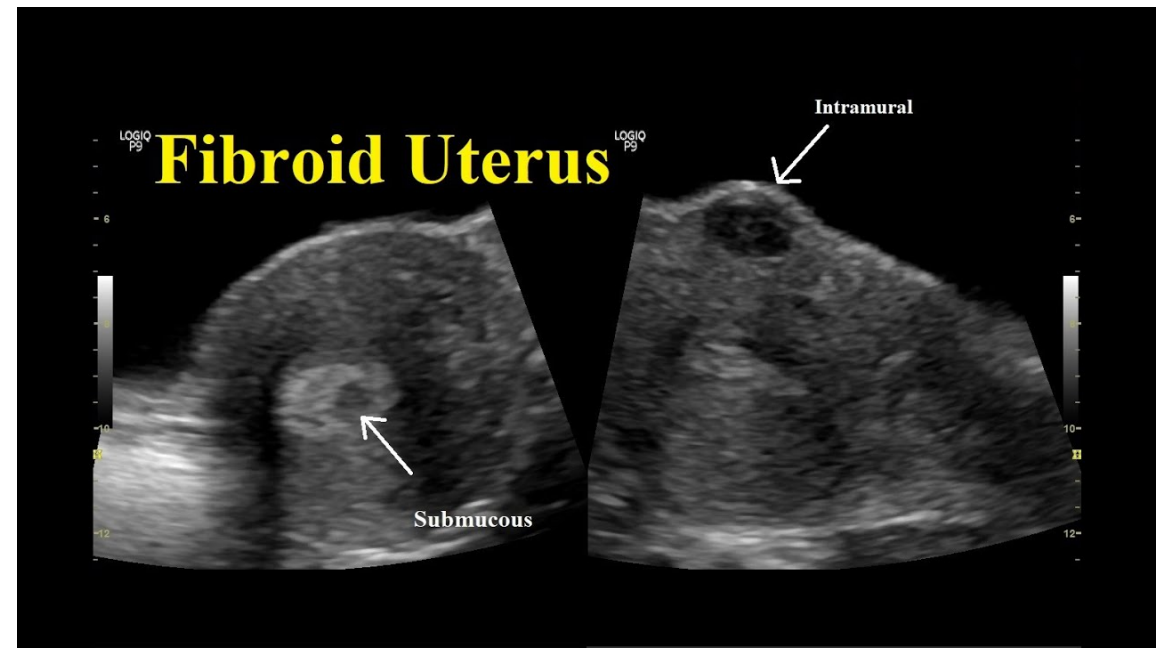

fig 3: A sonogram showing submucosal fibroid in a longitudnal view . 


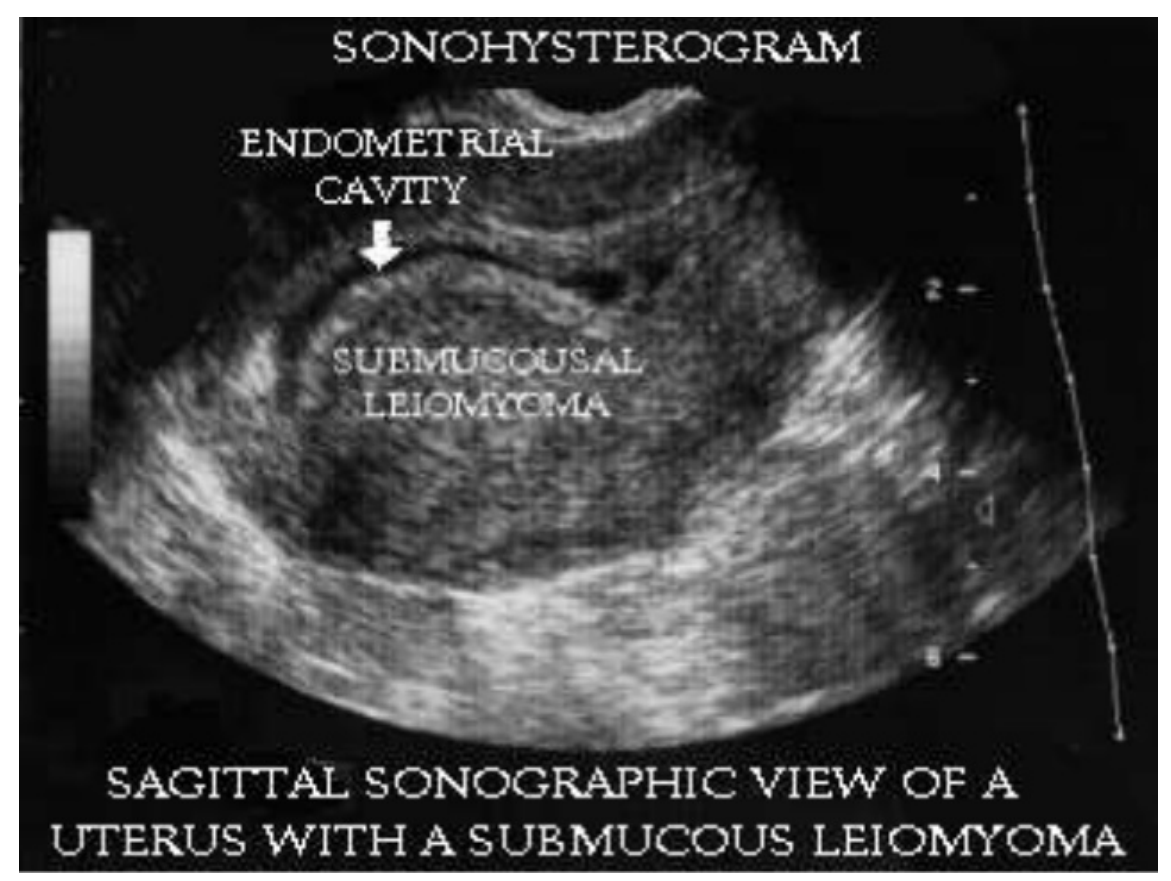

Fig 4: is showing all types of uterine leiomyomas and their positions

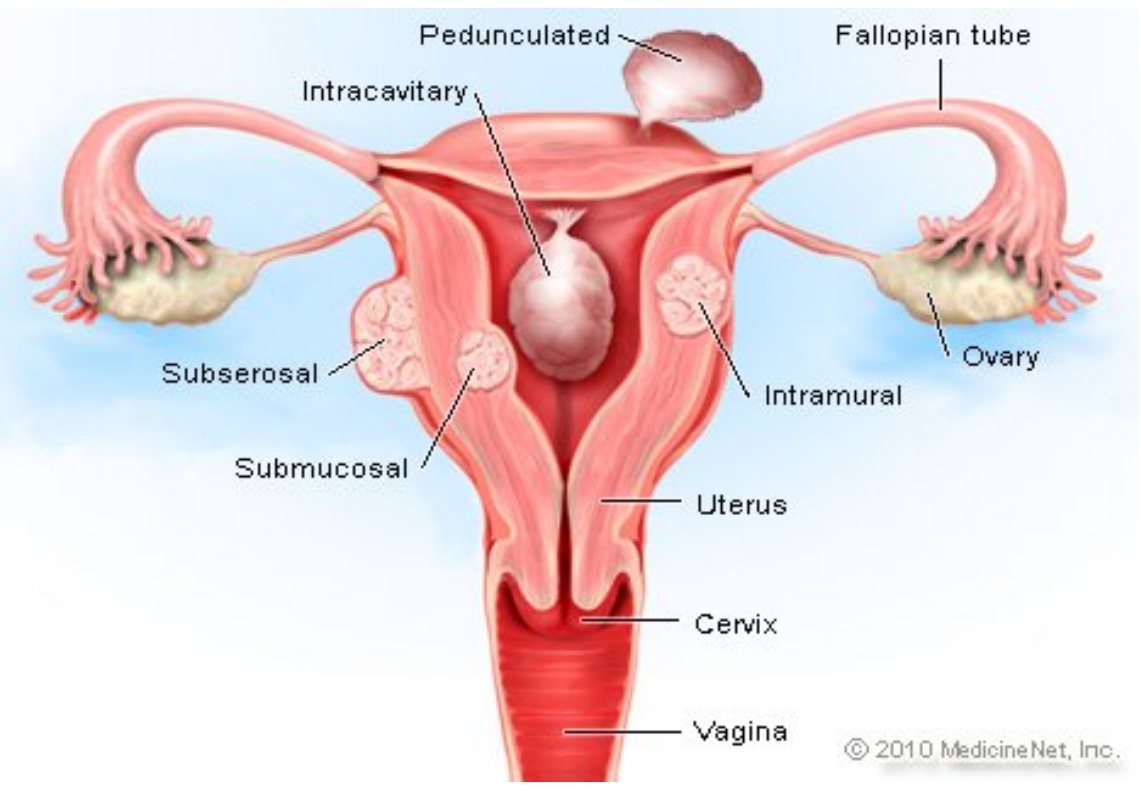

